\title{
Degrees of Freedom for Complex MIMO Multiple-Way Relay Channel
}

\author{
Xiao Chen, Yan Zhu, Xinxing Yin, Fangbiao Li and Zhi Xue \\ School of Electronic, Information and Electrical Engineering \\ Shanghai Jiaotong University \\ Shanghai, China \\ \{ chenxiao, topbestzy1983, yinxinxing, flyin2009, zxue\}@sjtu.edu.cn
}

\begin{abstract}
The amount of diversity or the number of degrees of freedom (DOF) can be increased by multiple antennas in wireless communication systems. In this paper, we study the DOF of multiple-input multiple-output (MIMO) multiple-way relay chomel. There are two groups of source nodes and several one relay node which are equipped with multiple antennas in this kind of channel. Each source node in one group exchanges independent message with the source node in the other group via the relay node. We first show the uper bound on the total DOF for this channel. Then we extend it to the complex MIMO multiple-way relay channel and present the corresponding upped bound.
\end{abstract}

Keywords: Degrees of Freedom (DOF) - capacity; multiple-way relay channel; MIMO

\section{Introduction}

Multiple antennas are important to improve the performance of wireless channel. In general, the spectral efficiency of MMMO channels is much higher than that of the conventional signal antem channels. Some research on MIMO channel [1-3], including the study of channel capacity and the design of communication schemes, illustrates a great improvement of performance.

There is recen interest in the degrees of freedom (DOF) of MIMO communication system. Tine, space, and trequency all offer DOF [4][5], spatial dimensions are especially interesting for how they may be accessed with distributed processing. Earlier work [6-8] by some researchers has determined the DOF of various multiuser MIMO systems. Then [9][10] derived the DOF for single-user point-to-point MIMO channel and two users MMMO multiple-access channel. [11] has shown that in the high signalto-noise radip (SNR) regime, the capacity of a channel with $m$ transmit, $n$ receive antennas and ri.d. Rayleigh faded gains between each antenna pair is given by:

$$
(S N R)=\min \{m, n\} \log S N R+O(1)
$$

Therefore, the number of DOF is the minimum of $m$ and $n$.

[12] considered the DOF of a multilayer distributed relay network. Based on [14-15] derived the upper bound on the DOF of the MIMO two-way X relay channel. In this paper, we focus on the MIMO system with relay, which is widely used for many kinds of networks. For example, in a cooperative multicell communication system with several base stations and several users via relays, the relays can help exchange information between them. Also, in ad hoc network and wireless mesh network, some users in one group exchange information with some users in the other group via relay 
nodes. Specially, this paper discusses the complex MIMO multiple-way relay channel. In this kind of channel, there are multiple relay nodes, each node is equipped with different number of antennas, and each group consists of different number of source nodes.

The remainder of this paper is organized as follows. Section 2 describes the MIMO multiple-way relay channel model. Section 3 shows the upper bound on the DOF of the MIMO multiple-way relay channel model. Section 4 extends Section 3 to more complex situation, and derives the corresponding upper bound on the DOF. Section 5 concludes the paper.

Notations: Boldface uppercase letters denote the matrices and boldface lowercase letters denote the vector. $(\square)^{H}$ and $\operatorname{Tr}(\square)$ are the Hermitian transpose and trace operators, respectively.

\section{MIMO Multiple-Way Channel Model}

A MIMO multiple-way relay channel is shown in Figure 1 of Section 6 . The channel consists of two groups of nodes and one relay node. There are L source hodes in each group. Each source node is equipped with $M$ antennas and the relay node is equipped with $\mathrm{N}$ antennas. Each node $A_{i}(i=1,2, \cdots, L)$ on the left side (group A) needs to send an independent message to each node $B_{j}(j=1,2, \cdots, L)$ on right side (group B) via the relay. So does the each source node on the group $B$

The total transmission is implemented in two phases. Firstly, in the multiple-access phase, the signals are transmitted to the relay from 11 the source nodes. The received signal at the relay are given by

$$
\mathbf{y}_{r}=\sum_{i=1}^{L} \mathbf{H}_{A_{i}, r} \mathbf{x}_{A_{i}}+\sum_{j=1}^{L} \mathbf{H}_{B_{j}, r} \mathbf{x}_{B_{j}}+\mathbf{n}_{r}
$$

Where $\mathbf{y}_{r}$ and $\mathbf{n}$ denote the $N \times 1$ feceived signal vector and the additive white Gaussian noise (AWGN) vector at the relay, respectively. $\mathbf{x}_{A_{i}}\left(\mathbf{x}_{B_{j}}\right)$ is the $M \times 1$ transmitted signal vector by source $A_{i}\left(Q_{B}\right)$ with the power constraint $\mathrm{E}\left(\operatorname{Tr}\left\{\mathbf{x}_{A_{i}} \mathbf{x}_{A_{i}}^{H}\right\}\right) \leq P$ $\left(\mathrm{E}\left(\operatorname{Tr}\left\{\mathbf{x}_{B_{j}} \mathbf{x}_{B_{j}}^{H}\right\}\right) \leq P \mathrm{H}_{A_{i}, r}\left(\mathbf{H}_{B_{j}, r}\right)\right.$ is the $N \times M$ channel matrix from source $A_{i}\left(B_{j}\right)$ to the relay. The entries of the channel matrix $\mathbf{H}_{A_{i}, r}\left(\mathbf{H}_{B_{j}, r}\right)$ and those of the noise $\mathbf{n}_{r}$ are independent and identically distributed (i.i.d.) zero-mean complex Gaussian random variables with unit ariance.

Secondly, in the broadcast phase, the relay node forms a new signal $\mathbf{x}_{r}$ and broadcasts it to all the source nodes. The received signals at the source nodes are given by

$$
\begin{aligned}
& \mathbf{y}_{A_{i}}=\mathbf{H}_{r, A_{i}} \mathbf{x}_{r}+\mathbf{n}_{A_{i}} \\
& \mathbf{y}_{B_{j}}=\mathbf{H}_{r, B_{j}} \mathbf{x}_{r}+\mathbf{n}_{B_{j}}
\end{aligned}
$$

Where $\mathbf{y}_{A_{i}}\left(\mathbf{y}_{B_{j}}\right)$ and $\mathbf{n}_{A_{i}}\left(\mathbf{n}_{B_{j}}\right)$ denote the $M \times 1$ received signal vector and the AWGN vector at the source node, respectively; $\mathbf{x}_{r}$ is the $N \times 1$ transmitted signal vector by the relay 
with the power constraint $\mathrm{E}\left(\operatorname{Tr}\left\{\mathbf{x}_{r} \mathbf{x}_{r}^{H}\right\}\right) \leq P ; \mathbf{H}_{r, A_{i}}\left(\mathbf{H}_{r, B_{j}}\right)$ is the $M \times N$ channel matrix from the relay to source node. It is assumed that $\mathbf{H}_{r, A_{i}}\left(\mathbf{H}_{r, B_{j}}\right)$ and $\mathbf{n}_{A_{i}}\left(\mathbf{n}_{B_{j}}\right)$ contain i.i.d. zero-mean complex Gaussian random variables with unit variance.

In this paper, we assume that perfect channel state information (CSI) is available at all source nodes and the relay. In addition, it is assumed that the source nodes and the relay node operate in full-duplex mode.

The total DOF of the above MIMO multiple-channel is defined as follow:

$d=\sum_{i=1}^{L} \sum_{j=1}^{L} d_{A_{i}, B_{j}}+\sum_{j=1}^{L} \sum_{i=1}^{L} d_{B_{j}, A_{i}}=\lim _{S N R \rightarrow \infty} \frac{R(S N R)}{\log (S N R)}$

Where $d_{A_{i}, B_{j}}\left(d_{B_{j}, A_{i}}\right)$ is the DOF from source node $A_{i}\left(B_{j}\right)$ to $B_{j}\left(A_{i}\right) R(S N R)$ is the sum rate as a function of $S N R$. Because the noise samples are assumed to have unit variance, $S N R$ is defined as $S N R \square P$

\section{An UpperBound on the DOF of the MMO Mulíple-Way Relay Channel}

In this section, an upper bound on the DOF of the MIMOMultiple-way relay channel is derived.

\section{Theorem 1:}

Consider a MIMO multiple-way elay channel with two groups of source nodes and one relay node. Each group consists of $\mathrm{L}$ source nodes, where each one is equipped with $\mathrm{M}$ antennas. And the relay node is equipped (ith $N$ antennas. The total number of DOF is upper bound by $2 \mathrm{~m}$ in $\{L M, N\}$ i.e.,

$d \leq 2 \min \{L M Q N\}$.

Proof: The network information flow of one direction is first considered, i.e., from $A_{i}$ to $B_{j}$ via the relay, as shown in Fig. 2 of Section 6.

In the first phase (cut),$A_{i}(i=1,2, \cdots, L)$ simultaneously transmit information to the relay. We assume that $A_{i}(i=1,2, \cdots, L)$ fully cooperate, then the channel essentially becomes a $L M \times N$ MIMO channel. From [13], we can derive the DOF that is $\min \{L M, N\}$. Similarly, in the second phase (cut 2) we can obtain the same result. Combining the two phase and the cul-set theorem[14], we can obtain the DOF of one direction

$$
\begin{aligned}
& \sum_{i=1} \sum_{j=1} d_{A_{i}, B_{j}} \leq \min \{\min \{L M, N\}, \min \{L M, N\}\} \\
& =\min \{L M, N\}
\end{aligned}
$$

For the other direction, the similar result can be derived as follow: 


$$
\begin{aligned}
& \sum_{j=1}^{L} \sum_{i=1}^{L} d_{B_{j}, A_{i}} \leq \min \{\min \{L M, N\}, \min \{L M, N\}\} \\
& =\min \{L M, N\}
\end{aligned}
$$

So

$$
d=\sum_{i=1}^{L} \sum_{j=1}^{L} d_{A_{i}, B_{j}}+\sum_{j=1}^{L} \sum_{i=1}^{L} d_{B_{j}, A_{i}} \leq 2 \min \{L M, N\}
$$

Proof completed.

Remark: We can notice that the factor 2 is needed since the assumption of full-duplex mode in this scheme. If the mode turns into half-duplex, the factor of 2 is not needed.

From theorem 1, we can see that when $N \leq L M$, the DOF of this channel is upper bounded by the twice the number of the relay node's antennas, which is therefore the bottleneck for the spectrum efficiency of the network.

\section{An Upper Bound on the DOF of the Complex MIMO Multiple-Way Relay Channel}

In practical application, only one relay node usual y cannot meet the demand, so more relay nodes should be needed to be deployed. We can extend the one relay node (Fig.1) to more relay nodes model (Fig.3). For convenience, call this lind of model complex MIMO multiple-way relay channel. In this section, we derive the upper bound on the DOF of the complex MIMO multiple-way relay channel.

\section{Theorem 2:}

Consider a complex MIMQ multiple-way relay channel with two groups of source nodes and $\mathrm{X}$ relay nodes. Each group consists of $\mathrm{L}$ source nodes, where each one is equipped with $\mathrm{M}$ antennas. And each relay node is equpped with $\mathrm{N}$ antennas. The total number of DOF is upper bound by $2 \mathrm{~m}$ in $\{L M, N\}$, i.e.,

$$
d \leq 2 \min \{L M, N\} .
$$

Proof: The process of proof is similar to theorem 1. The network information flow of one direction is also first considered. Then we cut this network into X+1 MIMO channel. Thus we have

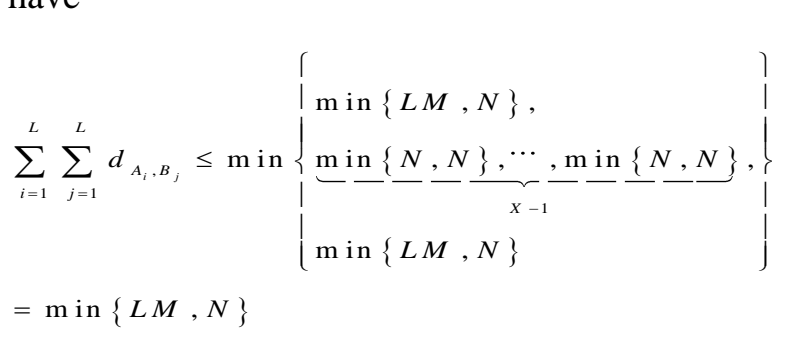

So

$d=\sum_{i=1}^{L} \sum_{j=1}^{L} d_{A_{i}, B_{j}}+\sum_{j=1}^{L} \sum_{i=1}^{L} d_{B_{j}, A_{i}} \leq 2 \mathrm{~min}\{L M, N\}$

Proof completed, 
Obviously, more relay nodes with the same number of antennas cannot change the upper bound on the DOF.

However, there is another more complex situation. From above situations, we notice that every source node has the same number of antennas $M$, so does every relay node. In the rest of this section, we will derive the upper bound on the DOF of the complex MIMO multiple-way relay channel with different antennas.

\section{Theorem 3:}

Consider a complex MIMO multiple-way relay channel with two groups of source nodes and $\mathrm{X}$ relay nodes. Group A consists of $\mathrm{L}_{1}$ source nodes where $A_{i}\left(i=1,2, \cdots, L_{1}\right)$ is equipped with $a_{i}$ antennas, and Group B consists of $\mathrm{L}_{2}$ source nodes where $B_{i}\left(j=1,2, \cdots L_{2}\right)$ is equipped with $b_{j}$ antennas. The relay node $\mathrm{k}$ is equipped with $c_{k}(k=1,2, \cdots, X)$ antennas. The total number of DOF is upper bound by:

$$
\begin{aligned}
& 2 \min \left\{\sum_{i=1}^{L_{1}} a_{i}, c_{1}, c_{2}, \cdots, c_{X}, \sum_{j=1}^{L_{2}} b_{j}\right\}, \text { i.e., } \\
& d \leq 2 \min \left\{\sum_{i=1}^{L_{1}} a_{i}, c_{1}, c_{2}, \cdots, c_{X}, \sum_{j=1}^{L_{2}} b_{j}\right\}
\end{aligned}
$$

Proof: The process of proof is similar to theorem 2 .

From theorem 3, we can see that the ninimum number of antennas among all the groups and relay nodes is the bottleneck for the spectrum efficiency of the network.

\section{Conclusions}

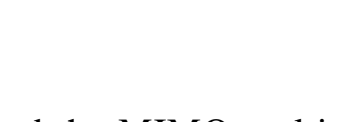

In this paper, we considered the MIMO multiple-way relay channel and the complex MIMO multiple-way relay channel. Then we obtained their corresponding upper bounds on the DOF, from which we can analyze the networks more effectively. By means of network coding and interference alignment, the upper bounds could be achieved, we will leave it to the future work..

\section{Graph}
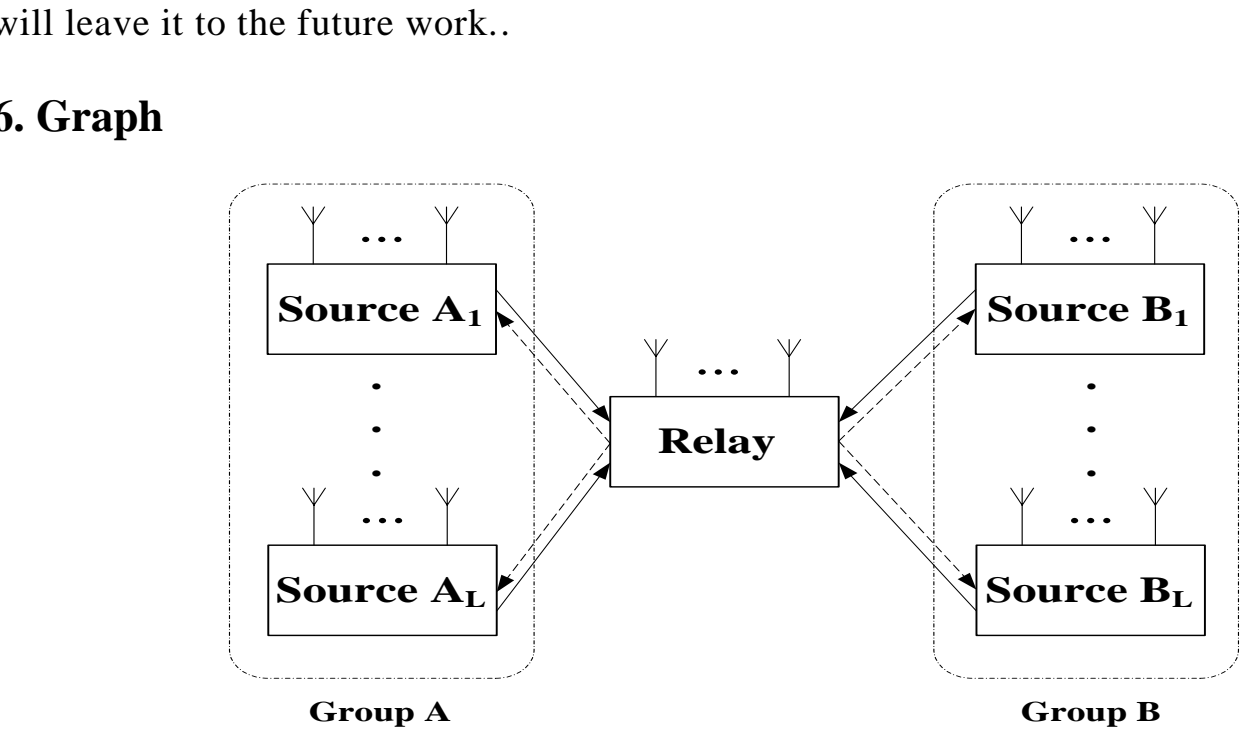

Figure 1. MIMO Multiple-way Relay Channel 


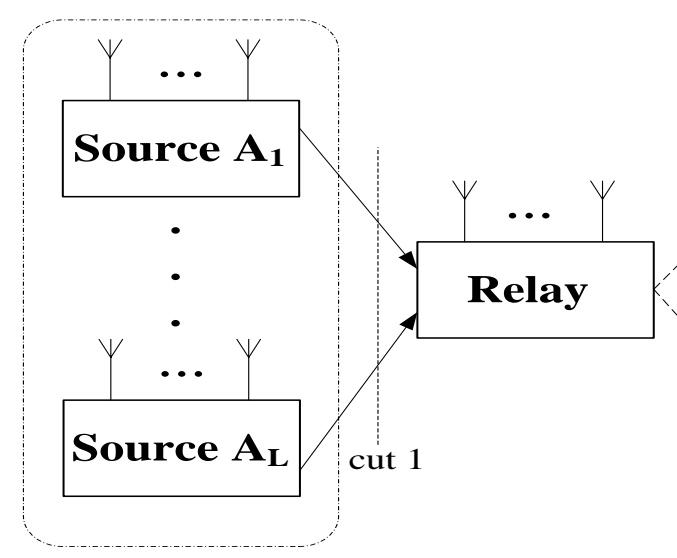

Group A

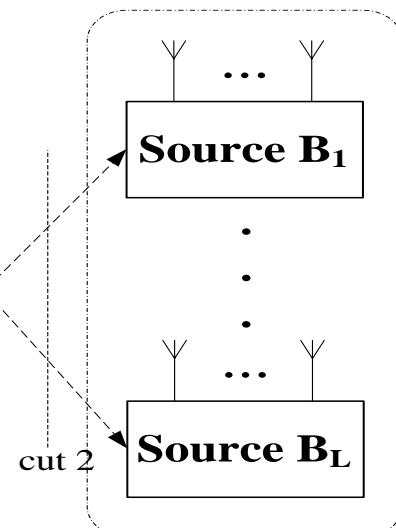

Group B

Figure 2. One Direction of Network Information Flow for the MIMO Multiple-way Relay Channel

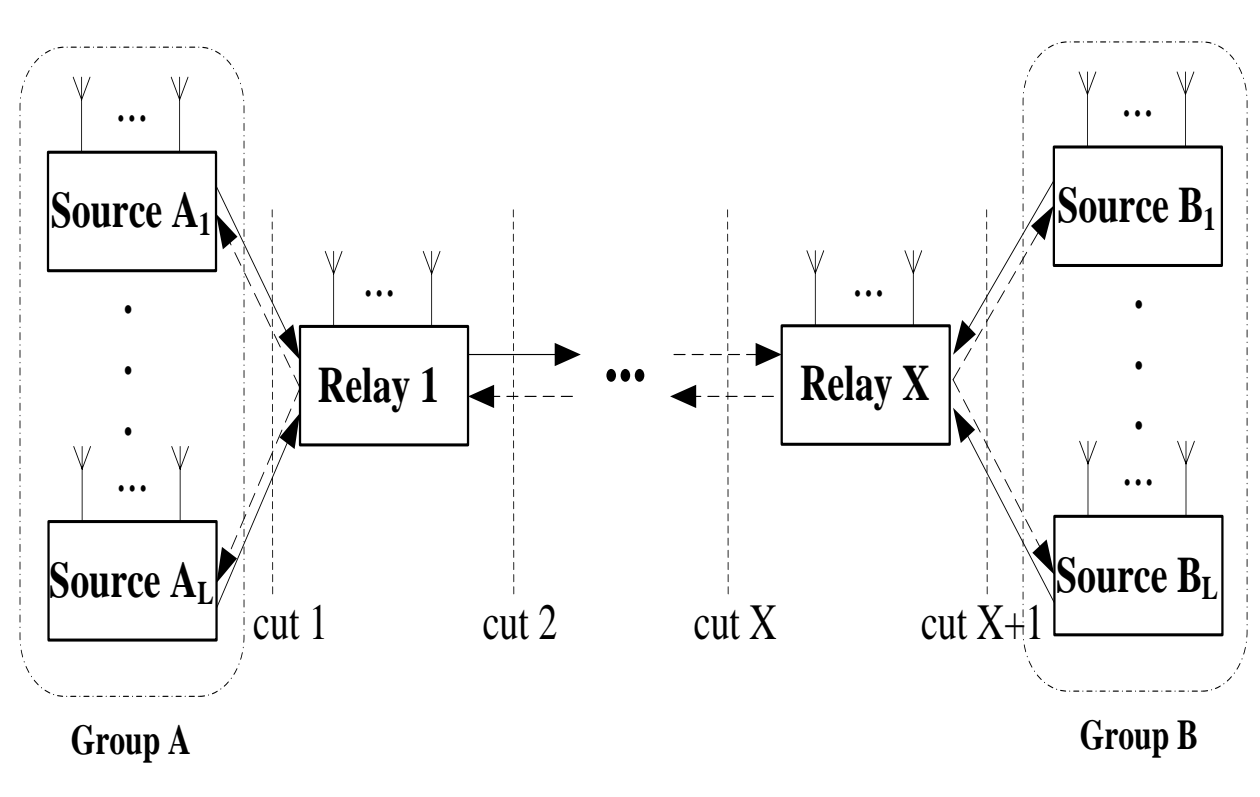

Figare 3. Complex MIMO Multiple-way Relay Channel

\section{Acknowledgements}

Xiao Chen and Yan Zhu contributed equally to this work and share first authorship. This work was supported in part by the Natural Science Foundation of China under Grant No. 60932003 , No. 61271220 and No. 61171173.

\section{References}

[1] I. E. Telatar, "Capacity of multi-antenna gaussian channels", European Trans. On Telecommunications, vol. 10, (1999), pp. 585-95.

[2] V. Tarokh, H. Jafarkhani and A. R. Calderbank, "Space-time block code from orthogonal designs", IEEE Trans. Info. Theory, vol. 45, (1999), pp. 1456-67.

[3] R. Heath Jr. and A. Paulraj, "Switching between multiplexing and diversity based on constellation distance", in Proc. of Allerton Conf. on Comm. Control and Comp., (2000). 
[4] A. Poon, R. Brodersen and D. N. C. Tse, "Degrees of freedom in multiple-antenna channels: A signal space approach", IEEE Trans. Inf.Theory, vol. 51, no. 2, (2006), pp. 523-536.

[5] L. Hanlen and T. Abhayapala, "Space-Time-Frequency Degrees of Freedom: Fundamental Limits for Spatial Information", arXiv:cs/0701056v1 [cs.IT], (2008).

[6] S. A. Jafar and M. Fakhereddin, "Degrees of freedom on the MIMO interference channel", in Proc. IEEE Int. Symp. Information Theory (ISIT), Seattle, WA, (2006), pp. 1452-1456.

[7] A. Høst-Madsen and A. Nosratinia, "The multiplexing gain of wireless networks", in Proc. IEEE Int. Symp. Information Theory (ISIT), (2005); Adelaide, Australia.

[8] H. Weingarten, S. Shamai (Shitz), and G. Kramer, "On the compound MIMO broadcast channel", in Proc. Annu. Information Theory and Applications Workshop UCSD, (2007); San Diego, La Jolla, CA.

[9] G. J. Foschini and M. J. Gans, "On limits of wireless communications in a fading environment when using multiple antennas", in Wireless Personal Commun. Norwell, MA: Kluwer Academic, (1998), pp. 311-335.

[10] D. N. C. Tse, P. Viswanath and L. Zheng, "Diversity-multiplexing tradeoff in multiple-access channels", IEEE Trans. Inf. Theory, vol. 50, no. 9, (2004); pp. 1859-1874.

[11] G. J. Foschini, "Layered space-time architecture for wireless communication in a fading environment whên using multi-element antennas", Bell Labs Technical Journal, vol. 1, no. 2, (1996); pp. 41-59.

[12] S. Borade, L. Zheng and R. Gallager, "Maximizing degrees of freedom in wireless networks", in Proc. 40th Annu. Allerton Conf. Communication, Control and Computing, Monticello, IL, (2003), pp. 561570.

[13]L. Zheng and D. Tse, "Diversity and multiplexing: A fundamental tradeoff in multiple-antenna channels", IEEE Trans. Inf. Theory, vol. 49, no. 5, (2003), pp. 1073-1096.

[14] T. Cover and A. Gamal, "Capacity theorems for the relay channel" IEEE Trans. Inf. Theory, vol. 25, no. 5, (1979), pp. 572-584.

[15]Z. Z. Xiang, M. X. Tao, et al., "Degrees of Freedom for MIMO Two-Way X Relay Channel", IEEE Transactions on Signal Processing, vol. 61, no. 7, (2013).

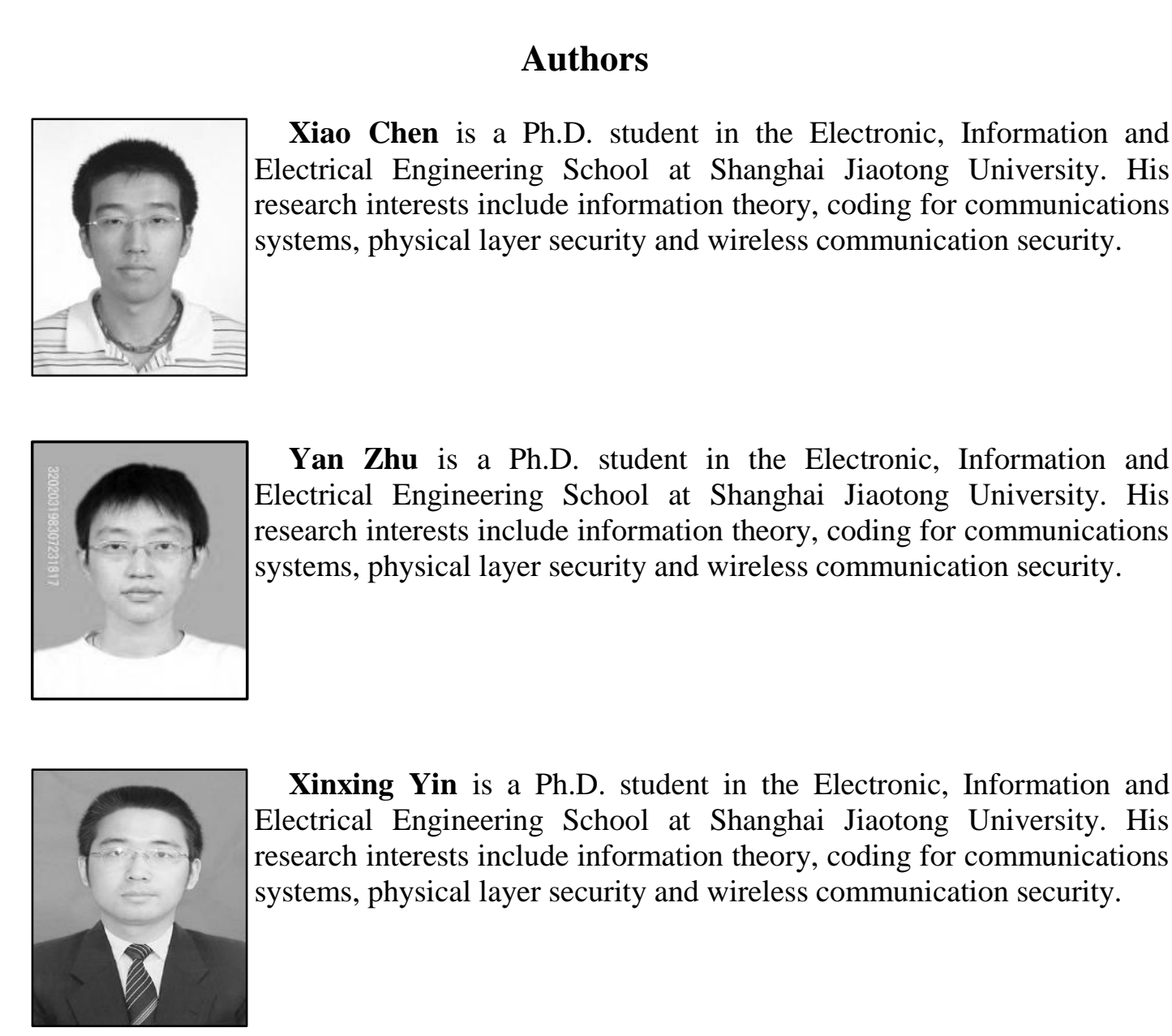




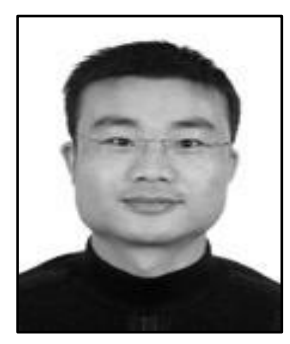

Fangbiao Li is a Ph.D. student in the Electronic, Information and Electrical Engineering School at Shanghai Jiaotong University. His research interests include information theory, coding for communications systems, physical layer security and wireless communication security.

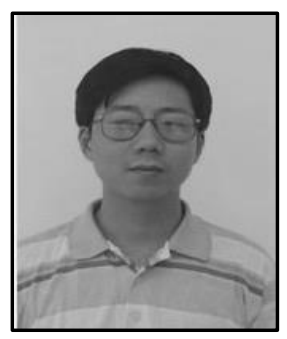

Zhi Xue received the B.Tech. degree from Shanghai Jiaotong University. He went to the United States for cooperation in scientific research at Bell Labs as a visiting scholar in 1997. Then he received Ph. $\widehat{D}$. degree from Shanghai Jiaotong University. Where he is an Professor and Doctoral Tutor. Currently, he is the vice president of Information Security Engineering School in Shanghai Jiaotong University. His research interests include information theory coding for communications systems, physical layer security and ireless communication security.

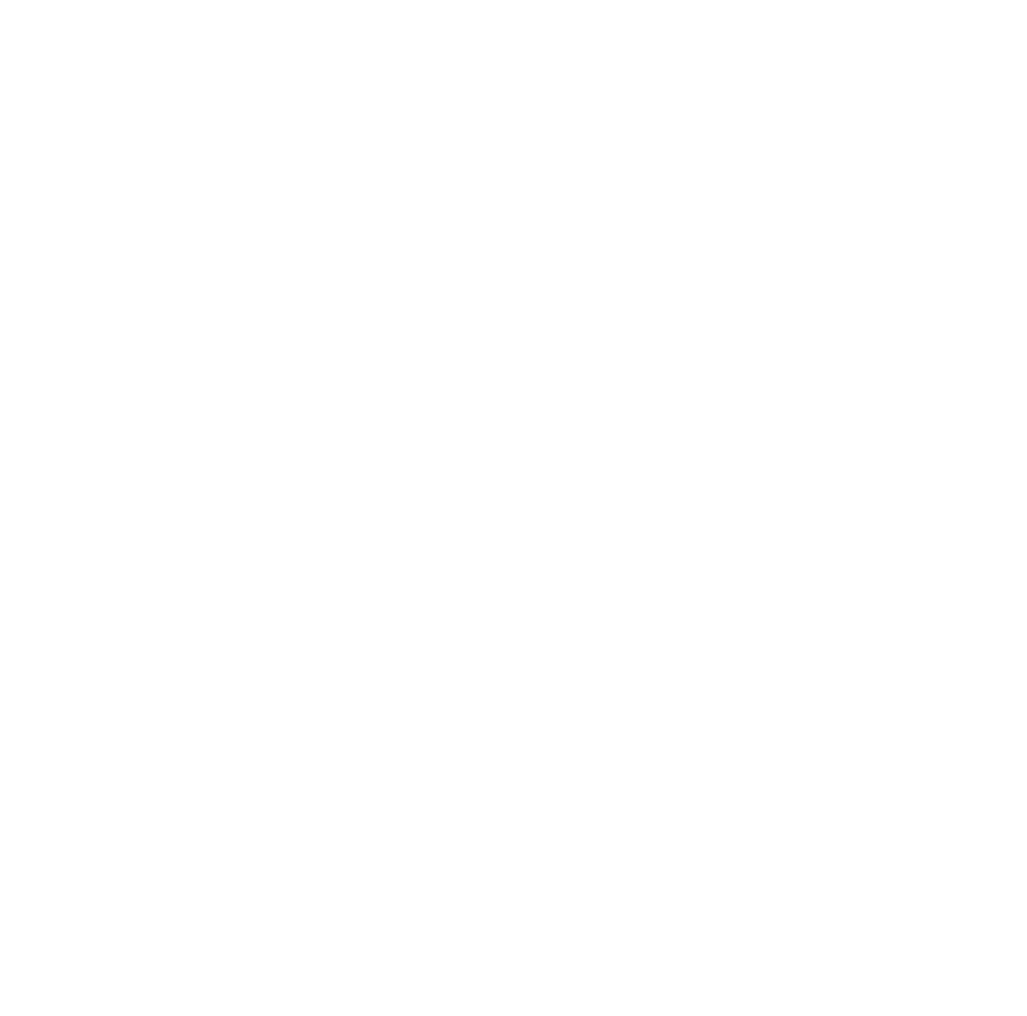

